\title{
Análisis comparativo entre la calidad de vida del paciente trasplantado renal y el paciente en hemodiálisis
}

\author{
Mónica Romero-Reyes ${ }^{1}$, Ana Moreno-Egea ${ }^{1}$,Victoria Eugenia Gómez-López ${ }^{2}$, Mateo Alcántara-Crespo ${ }^{2}$ \\ Rodolfo Crespo-Montero ${ }^{1,2,3}$ \\ ${ }^{1}$ Departamento de Enfermería. Facultad de Medicina y Enfermería. Universidad de Córdoba.España \\ ${ }^{2}$ Servicio de Nefrología. Hospital Universitario Reina Sofía de Córdoba. España \\ ${ }^{3}$ Instituto Maimónides de Investigación Biomédica de Córdoba. España
}

\section{Resumen}

Objetivo: El objetivo del presente estudio fue comparar la calidad de vida relacionada con la salud del paciente trasplantado renal frente al paciente en hemodiálisis.

Material y Método: Estudio observacional comparativo por emparejamiento (1:2) en 240 pacientes (80 en hemodiálisis y 160 trasplantados renales). El muestreo fue no probabilístico intencionado por emparejamiento. El emparejamiento se realizó por: edad, sexo y comorbilidad. Se utilizó el cuestionario KDQOL-SF para analizar calidad de vida relacionada con la salud.

Resultados: Los pacientes tenían una edad de $59,80 \pm 12,45$ años. Los pacientes trasplantados presentaron mejores puntuaciones, con diferencias significativas, en todas las dimensiones de las Escalas específicas para las enfermedades renales, excepto en el Apoyo Social. De igual forma, puntuaron mejor con diferencias significativas, los pacientes trasplantados en todas las dimensiones en el cuestionario SF-36. Los pacientes diabéticos trasplantados puntuaron peor en las dimensiones Carga de la enfermedad renal y Dolor; y los pacientes diabéticos en diálisis, en el Rol emocional. En el grupo de trasplantados,

\section{Correspondencia:}

Mónica Romero Reyes

Email: monica.romero.reyes@gmail.com
Como citar este artículo: Crespo M, Crespo-Montero R. splantado renal y el paciente en hemodiálisis.

Enferm Nefrol. 2021 Abr-Jun;24(2):129-38

\section{Comparative analysis between the quality of life of the renal transplant patient and the haemodialysis patient}

las mujeres puntuaron peor de forma significativa, en las dimensiones Listado de síntomas/problemas, Efectos de la enfermedad, Dolor y Vitalidad; y el grupo de pacientes en hemodiálisis, las mujeres puntuaron peor en la Función física.

Conclusiones: Emparejados por edad, sexo y diabetes, los pacientes trasplantados renales presentan mejor calidad de vida relacionada con la salud que los pacientes en hemodiálisis, tanto en las Escalas específicas para la enfermedad renal como en las dimensiones del SF-36. En ambos grupos, los pacientes diabéticos presentan peor calidad de vida que los no diabéticos.

PALABRAS CLAVE: calidad de vida relacionada con la salud; enfermedad renal crónica avanzada; trasplante renal; hemodiálisis.

\section{Abstract}

Objective: The aim of the present study was to compare the health-related quality of life of the renal transplant patient versus the haemodialysis patient. 
Material and Method: Matched (1:2) observational comparative study in 240 patients ( 80 on haemodialysis and 160 renal transplant recipients). Sampling was nonprobabilistic purposive sampling. Matching was done by age, sex, and comorbidity. The KDQOL-SF questionnaire was used to analyse health-related quality of life.

Results: Patients had a mean age of $59.80 \pm 12.45$ years. Transplant patients had better scores, with significant differences, in all dimensions of the specific scales for renal diseases, except in "social support". Similarly, transplant patients scored better, with significant differences, in all dimensions of the SF-36 questionnaire. Transplant diabetic patients scored worse on the dimensions "burden of kidney disease" and "pain", and diabetic patients on dialysis on "emotional role". In the transplant group, women scored significantly worse on the dimensions "list of symptoms/problems", "effects of the disease", "pain" and "vitality"; and in the haemodialysis group, women scored worse on "physical function".

Conclusions: Matched for age, sex and diabetes, renal transplant patients present better health-related quality of life than haemodialysis patients, both in the "renal disease-specific scales" and in the SF-36 dimensions. In both groups, diabetic patients have worse quality of life than non-diabetic patients.

KEYWORDS: health-related quality of life; advanced chronic kidney disease; renal transplantation; haemodialysis; kidney transplantation.

\section{Introducción}

La Enfermedad Renal Crónica Avanzada (ERCA) ha aumentado progresivamente a lo largo de los años $y_{\text {, }}$ consecuentemente, el número de pacientes que necesitan terapia renal sustitutiva (TRS), alcanzando cifras consideradas como epidémicas ${ }^{1,2}$. En la actualidad, la incidencia se sitúa en 127 pacientes por millón de población, con un aumento progresivo de los pacientes mayores de 65 años $^{3}$. Son varios los factores que influyen en el incremento de pacientes con ERCA: envejecimiento de la población, incremento de la patología de diabetes mellitus (DM) ${ }^{4}$ y otros factores como un aumento del síndrome metabólico por obesidad y sedentarismo entre otros. Según el Registro de la Sociedad Española de Nefrología, en nuestro país la incidencia de la ERCA es de 145 pacientes por millón de población, teniendo el $25 \%$ la DM como enfermedad renal primaria. En cuanto a la prevalencia, se sitúa en 1320 pacientes por millón de población, estando el $54,5 \%$ de los pacientes con un Trasplante Renal (TR) funcionante, el 40,3\% en hemodiálisis (HD) y el $5 \%$ en diálisis peritoneal (DP), como TRS ${ }^{5}$. Al incremento progresivo de la edad de estos pacientes, se le suma también un aumento de patologías añadidas o comorbilidad, con un alto porcentaje de diabéticos, originando un incremento de ingresos hospitalarios ${ }^{6}, y$ condicionando todo ello, peor percepción de su calidad de vida ${ }^{7}$.

La Organización Mundial de la Salud definió Salud como un completo estado de bienestar físico, mental y socia ${ }^{8}$. Este concepto salud está relacionado con la definición posterior del concepto Calidad de Vida Relacionada con la Salud (CVRS), que hace referencia a la percepción de salud del individuo, recogiendo aspectos tanto subjetivos como objetivos?. Los pacientes con ERCA presentan peor CVRS que la población general sana, con disminución de la misma desde la entrada en prediálisis ${ }^{10}$, acentuándose esta disminución de calidad de vida cuando los pacientes están con TRS en programas de diálisisis ${ }^{11}$. Así, los pacientes en HD presentan bajas puntuaciones en casi todas las dimensiones que se evalúan en la CVRS, estando estas bajas puntuaciones relacionadas con la edad, sexo, comorbilidad, técnica de diálisis utilizada, estado cognitivo-emocional, creencias y experiencias vividas ${ }^{12}$.

En este sentido, utilizando como referencia el Cuestionario de Enfermedad del Riñón y Calidad de Vida KDQOL-SF ${ }^{\mathrm{T} M} 36$, las dimensiones más afectadas de los pacientes en HD suelen ser la Carga de la enfermedad renal, Sueño, Salud general, Vitalidad, Función física y Rol físico ${ }^{10}$; además, esta peor percepción de la CVRS de los pacientes en HD está muy relacionada con el componente ansiedad/depresión ${ }^{13}$.

Sin embargo, los pacientes con ERCA con un TR funcionante presentan mejor CVRS en cuanto a aspectos generales y síntomas específicos de la enfermedad, menor riesgo de mortalidad y disminución de los eventos cardiovasculares ${ }^{14}$; por lo que obviamente, se recomienda que la TRS de elección en los pacientes con ERCA debe ser el TR ${ }^{15,16}$.

De hecho, en algunos estudios en los que se compara la CVRS del paciente en HD, con los pacientes con un TR funcionante, estos últimos presentan mejores puntuaciones en la mayoría de las dimensiones que componen los cuestionarios de la CVRS, asemejándose a la de 
la población general ya que el paciente en HD cuando recibe un injerto renal va recuperando en gran medida la salud perdida ${ }^{17,18}$. Si a esto añadimos que el paciente con ERCA tras ser trasplantado deja de depender de diálisis, disponiendo de más tiempo para volver a sus actividades cotidianas, es lógico pensar que recibir un injerto renal funcionante repercuta de forma positiva en su bienestar a largo plazo ${ }^{19}$.

Sin embargo, los estudios que comparan la CVRS entre diferentes TRS suelen tener un sesgo de selección importante, pues a menudo comparan poblaciones diferentes en cuanto a variables sociodemográficas y de comorbilidad.

Por este motivo hemos diseñado el presente estudio para comparar la CVRS entre pacientes en HD y trasplantados renales "emparejados" por similares características clínicas o covariables como son el sexo, la edad y la comorbilidad (diabetes).

\section{Objetivos}

Comparar la calidad de vida de los pacientes trasplantados de riñón frente a pacientes en HD mediante un estudio de emparejamiento.

Objetivos secundarios:

- Determinar las variables más influyentes en la CVRS en el paciente con TR.

- Precisar las variables más determinantes en la CVRS en el paciente en HD.

\section{Material y Método}

\section{Ámbito del estudio}

El estudio se ha llevado a cabo en el Servicio de Nefrología del Hospital Universitario Reina Sofía de Córdoba (HURS). El estudio se llevó los a cabo entre noviembre de 2019 y mayo de 2020.

\section{Diseño del estudio}

Se realizó un estudio observacional descriptivo comparativo por emparejamiento.

\section{Población y muestra}

- Selección de los participantes: la población de estudio fueron los pacientes con ERCA en HD y trasplantados renales dependientes del Servicio de Nefrología del HURS.
- Criterios de inclusión: pacientes de ambos sexos, mayores de 18 años, con TR funcionante que se pudieran emparejar con los pacientes del programa de HD, sin alteraciones cognitivas que le impidieran responder a los cuestionarios. Para ello se utilizó la base de datos de pacientes en HD del Servicio de Nefrología de Córdoba, seleccionando a aquellos pacientes que se les había analizado la CVRS en los últimos 6 meses $(n=170)$. Partiendo de esta muestra y mediante la asignación de las covariables de emparejamiento se procedió al mismo en la base de datos de los correspondientes pacientes trasplantados renales. Se realizó un emparejamiento o macheo de 1 a 2 (1 paciente en HD por 2 con TR), para mantener la proporción entre ambos grupos de pacientes en la provincia de Córdoba. Las covariables de emparejamiento fueron: edad, sexo y comorbilidad (diabetes).

- Tipo de muestreo: se realizó un muestreo no probabilístico intencionado por emparejamiento, que dependió del número final de pacientes que se consiguió emparejar. Se consiguió una muestra final de 240 pacientes: 80 en HD y 160 con TR (1:2).

\section{Definición de variables}

La variable de resultado fue la CVRS. Otras variables añadidas en el estudio han sido: edad, sexo, comorbilidad, tiempo en HD, tiempo en TR.

\section{Instrumentos de medida}

Para determinar la CVRS se utilizó el cuestionario validado KDQOL-SF, que es una adaptación del cuestionario SF-36 para pacientes con ERCA ${ }^{20}$. En los pacientes con TR no fueron cumplimentadas las cuestiones relativas a la satisfacción del paciente y actitud del personal de diálisis. Para todas las dimensiones, 100 es la mejor puntación de CVRS. Para el análisis de la comorbilidad se utilizó el Índice de Comorbilidad de Charlson (ICC).

\section{Recogida de datos}

Los datos se iban a recoger en la propia consulta de TR del Servicio de Nefrología, en función de las citas programadas de los pacientes en esta consulta. Pero ante la situación extraordinaria por la COVID-19, los datos tuvieron que ser obtenidos mediante llamada telefónica. En todo momento, se preservó la confidencialidad de los datos del paciente. La entrevista se realizó a aquellos pacientes que, tras ser informados del estudio y lectura del consentimiento informado, aceptaron participar en el mismo. Las llamadas telefónicas se realizaron en días laborables y en horario que no ocasionase molestias al paciente. 


\section{Análisis estadístico}

Para las variables cualitativas se utilizó una distribución de frecuencias, mientras que para las variables cuantitativas la media \pm la desviación estándar de la media 0 la mediana y rango intercuartílico, según los datos siguieran o no una distribución normal. Para comparar las variables cuantitativas de los grupos emparejados se utilizó la T de Student para muestras independientes o la U de Mann-Whitney, según siguieran o no una distribución normal. Se realizaron correlaciones con el test de Pearson para establecer asociaciones entre las diferentes variables. Se aceptó significación estadística para $p<0,05$. Los datos se analizaron con el paquete estadístico SPSS 18.0 para Windows.

\section{Aspectos Éticos y Legales}

La información obtenida ha sido tratada conforme a la actual Ley Orgánica 3/2018, de 5 de diciembre, de "Protección de Datos Personales y garantía de los derechos digitales", y con el único fin del desarrollo de esta investigación. La participación en el estudio fue en todo momento de carácter voluntaria y anónima, respetando en cualquier punto de su desarrollo que cualquier usuario decidiera abandonar la entrevista.

El estudio contó con el informe favorable del Comité de Ética e Investigación del HURS (TFGCVRS-2020).

\section{Resultados}

Se estudiaron 240 pacientes ( 80 en HD y 160 con TR) con una edad media de 59,80 $\pm 12,457$ años; sin diferencias significativas entre la edad de los pacientes en HD y con TR. En total, entre ambos grupos, encontramos 165 varones $(69 \%)$ y 75 mujeres $(31 \%)$. Del total de pacientes, 36 (15\%) eran diabéticos. La media de ICC para la muestra fue de $4,75 \pm 2,497$ puntos.

Los pacientes en HD llevaban $64,1 \pm 64$ meses en diálisis y los pacientes con TR llevaban $131,1 \pm 104,6$ meses con un injerto renal funcionante.

Del total de pacientes en HD, 55 (69\%) se dializaban a través de una Fístula Arteriovenosa autóloga (FAVa) y $25(31 \%)$ lo hacían a través de un Catéter venoso central tunelizado (CATt).

En cuanto a los valores de las variables por las se emparejaron los pacientes se pueden observar en la Tabla 1.
En cuanto a la comparación entre el ICC entre ambos grupos de pacientes, encontramos un ICC de 5,74 $\pm 2,98$ en los pacientes en HD frente a $3,91 \pm 1,57$ puntos, en los pacientes con TR, siendo estas diferencias estadísticamente significativas $(p<0,001)$.

Tabla 1. Valores de las covariables por las que se ajustaron ambos grupos.

\begin{tabular}{l|l|l}
\hline & HD (80 pacientes) & TR (160 pacientes) \\
\hline Edad & $59,64 \pm 12,5$ años & $59,88 \pm 12,4$ años \\
\hline Sexo & 25 mujeres (31\%) & 50 mujeres $(31 \%)$ \\
& 55 hombres (69\%) & 110 hombres $(69 \%)$ \\
\hline Diabéticos & $12(15 \%)$ & $24(15 \%)$ \\
\hline
\end{tabular}

Cuando se compararon las puntuaciones medias entre el grupo de pacientes en HD y TR, se encontraron diferencias estadísticamente significativas en todas las dimensiones de las Escalas Específicas para Enfermedades Renales, excepto en el Apoyo social, y en todas las dimensiones del SF-36, tal como puede apreciarse en la Tabla 2.

En la figura 1 podemos observar las diferencias de puntuaciones entre los pacientes en HD y en TR, para el cuestionario genérico de CVRS SF-36, siendo las diferencias más acusadas en las dimensiones Rol físico, Rol emocional y Función social.

Respecto al análisis por separado de ambos grupos, podemos destacar en los pacientes con TR que al comparar la CVRS entre hombres y mujeres, encontramos que las mujeres puntuaron peor en casi todas las dimensiones del KDQOL-SF, aunque las diferencias solo fueron significativas en las dimensiones Listado de síntomas/ problemas, Efectos de la enfermedad renal, Dolor y Vitalidad, como observarse en la Tabla 3.

Cuando se comparó el ICC entre pacientes diabéticos y no diabéticos con TR se encontraron diferencias estadísticamente significativas $(5,39 \pm 1,3$ frente a $3,54 \pm 1,4$, $p<0,001)$. De igual forma, puntuaron peor, con diferencias significativas los diabéticos en las dimensiones Carga de la enfermedad renal $(97,13 \pm 6,3$ frente a $90,34 \pm 19,5, p<0,01)$ y Dolor $(92,91 \pm 13,7$ frente a $83,45 \pm 22,4, p<0,01)$.

Además, se encontró correlación significativa entre el tiempo que llevaban trasplantados con los Efectos de la enfermedad renal $(r=0,163, p<0,05)$, la Función física $(r=0,161, p<0,05)$ y el ICC $(r=-0,238, p<0,05)$. El ICC 
Tabla 2. Comparación entre pacientes en HD y con TR, para las dimensiones de los cuestionarios KDQOL-SF.

\begin{tabular}{|c|c|c|c|}
\hline Escalas específicas para enfermedades renales & HD & TR & Valor de $\mathbf{p}$ \\
\hline Listado de síntomas/problemas & $73,7 \pm 16,9$ & $91,2 \pm 9,4$ & $p<0,001$ \\
\hline Efectos de la enfermedad renal & $59,7 \pm 22,2$ & $94,5 \pm 8$ & $\mathrm{p}<0,001$ \\
\hline Carga de la enfermedad renal & $40,4 \pm 26,2$ & $91,4 \pm 18,3$ & $p<0,001$ \\
\hline Situación laboral & $21,6 \pm 28,4$ & $37,5 \pm 33,6$ & $\mathrm{p}<0,001$ \\
\hline Función cognitiva & $79,2 \pm 19,3$ & $98,4 \pm 6,3$ & $\mathrm{p}<0,001$ \\
\hline Calidad de las relaciones sociales & $82,0 \pm 20,5$ & $95,1 \pm 12,2$ & $p<0,001$ \\
\hline Función sexual & $54,7 \pm 43,4$ & $97,9 \pm 7,9$ & $\mathrm{p}<0,001$ \\
\hline Sueño & $60,0 \pm 25,4$ & $75,4 \pm 20,5$ & $\mathrm{p}<0,001$ \\
\hline Apoyo social & $85,6 \pm 59,2$ & $93,4 \pm 15,2$ & NS \\
\hline Ítems del SF-36 & HD & $\mathbf{T R}$ & Valor de $\mathbf{p}$ \\
\hline Función física & $56,1 \pm 29,5$ & $74,9 \pm 26,3$ & $\mathrm{p}<0,001$ \\
\hline Rol físico & $26,6 \pm 37,6$ & $66,7 \pm 38,6$ & $\mathrm{p}<0,001$ \\
\hline Dolor & $67,8 \pm 32,0$ & $84,9 \pm 21,5$ & $p<0,001$ \\
\hline Salud general & $40,1 \pm 18,8$ & $59,3 \pm 21,3$ & $p<0,001$ \\
\hline Bienestar emocional & $63,9 \pm 25,0$ & $78,3 \pm 19,3$ & $p<0,001$ \\
\hline Rol emocional & $61,3 \pm 47,0$ & $87,3 \pm 30,6$ & $p<0,001$ \\
\hline Función social & $65,6 \pm 32,7$ & $91,9 \pm 19,4$ & $p<0,001$ \\
\hline Vitalidad & $47,7 \pm 26,8$ & $74,5 \pm 20,1$ & $p<0,001$ \\
\hline
\end{tabular}

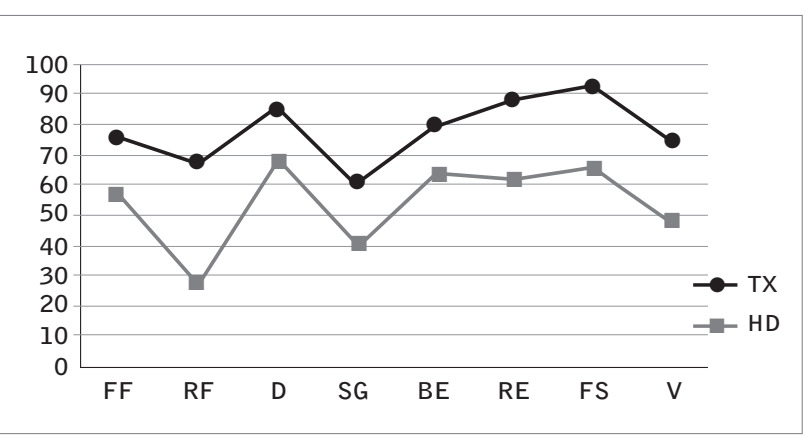

Figura 1. Valores medios de las dimensiones del Cuestionario SF36 entre ambos grupos.

FF: Función Física, RF: Rol Físico, D: Dolor, SG: Salud General, BE: Bienestar Emocional, RE: Rol Emocional, FS: Función Social, V: Vitalidad.

se correlacionó también con Función física $(r=-0,250$, $p<0,05)$ y la edad $(r=0,510, p<0,01)$. En los pacientes con TR, la edad se relacionó con las dimensiones Listado de síntomas/problemas $(r=-0,188, p<0,05)$ y Situación laboral $(r=-0,257, p<0,01)$, de las Escalas específicas para enfermedades renales; y con Función física $(r=-0,338, p<0,01)$, Dolor $(r=-0,270, p<0,01)$ y Vitalidad $(r=-0,170, p<0,05)$, del cuestionario genérico SF-36.
En cuanto a los pacientes en HD, cuando se comparó el ICC entre pacientes diabéticos y no diabéticos, se encontraron diferencias estadísticamente significativas $(6,75 \pm 1,2$ frente a $5,55 \pm 3,1, p<0,001)$. De igual for$\mathrm{ma}$, puntuaron peor, con diferencias significativas, los diabéticos en la dimensión Rol emocional frente a los no diabéticos $(30,55 \pm 43,7$ frente a $66,66 \pm 45,7, p<0,05)$. Cuando se compararon las puntuaciones del cuestionario KDQOL-SF, entre hombres y mujeres en los pacientes en HD, las mujeres puntuaron peor de forma significativa en la dimensión Función física $(63 \pm 25$ frente a $40,8 \pm 31, p<0,001)$.

En cuanto a los pacientes en HD, la edad sólo se relacionó con la dimensión Función física ( $r=-0,294, p<0,01)$, del SF-36. También se encontró correlación significativa entre el tiempo que llevaban en diálisis con los Efectos de la enfermedad renal $(r=-0,339, p<0,01)$, la Función sexual $(r=-0,259, p<0,05)$, Sueño $(r=-0,359$, $p<0,01)$ y Salud general $(r=-0,258, p<0,05)$. El ICC se correlacionó con la edad $(r=0,352, p<0,01)$.

Finalmente, cuando comparamos la CVRS entre los pacientes que se dializaban con una FAVa con los que se dializaban mediante un CATt, encontrando peores pun- 
Tabla 3. Comparación entre hombres y mujeres para las dimensiones del cuestionario KDQOL-SF en los pacientes con TR.

\begin{tabular}{|c|c|c|c|}
\hline Escalas específicas para enfermedades renales & Hombres & Mujeres & Valor de $\mathbf{p}$ \\
\hline Listado de síntomas/problemas & $92,5 \pm 8,6$ & $88,9 \pm 10,4$ & $p<0,01$ \\
\hline Efectos de la enfermedad renal & $95,65 \pm 6,6$ & $92,03 \pm 9,5$ & $P<0,05$ \\
\hline Carga de la enfermedad renal & $91,5 \pm 18,7$ & $91,1 \pm 17,6$ & NS \\
\hline Situación laboral & $40,4 \pm 35,5$ & $31,0 \pm 28,3$ & NS \\
\hline Función cognitiva & $98,7 \pm 4,7$ & $97,7 \pm 8,8$ & NS \\
\hline Calidad de las relaciones sociales & $94,8 \pm 13,9$ & $95,9 \pm 9,1$ & NS \\
\hline Función sexual & $98,6 \pm 6,6$ & $96,3 \pm 10,2$ & NS \\
\hline Sueño & $77,5 \pm 19,9$ & $70,7 \pm 21,3$ & NS \\
\hline Apoyo social & $94,8 \pm 13,7$ & $99,3 \pm 17,8$ & NS \\
\hline Ítems del SF-36 & Hombres & Mujeres & Valor de $\mathbf{p}$ \\
\hline Función física & $77,2 \pm 25,7$ & $70,0 \pm 27,1$ & NS \\
\hline Rol físico & $67,0 \pm 38,6$ & $66 \pm 39,0$ & NS \\
\hline Dolor & $87,9 \pm 19,6$ & $78,1 \pm 24,1$ & $p<0,01$ \\
\hline Salud general & $60,2 \pm 21,7$ & $57,3 \pm 20,6$ & NS \\
\hline Bienestar emocional & $80,1 \pm 19,1$ & $74,5 \pm 19,4$ & NS \\
\hline Rol emocional & $88,2 \pm 29,1$ & $85,3 \pm 33,8$ & NS \\
\hline Función social & $92,4 \pm 19,5$ & $90,8 \pm 19,2$ & NS \\
\hline Vitalidad & $77,3 \pm 19,2$ & $68,5 \pm 21,0$ & $p<0,01$ \\
\hline
\end{tabular}

tuaciones en los pacientes dializados mediante CATt en varias de las dimensiones del KDQOL-SF, aunque las diferencias solo fueron significativas en las dimensiones Efectos de la enfermedad renal, Función sexual y Rol físico.

\section{Discusión}

Dada la influencia que el TR ha demostrado tener sobre la mejora de la CVRS en los pacientes con ERCA previamente en diálisis, planteamos el presente estudio para comprobar si las diferencias persistían tras emparejar a los pacientes por edad, sexo y ser o no diabético en ambas poblaciones, ya que estas variables han mostrado tener una influencia directa en la CVRS en los pacientes con ERCA ${ }^{21}$.

Las puntuaciones, tanto del cuestionario de las Escalas específicas para enfermedades renales como de los ítems del SF-36, han sido mejores en los pacientes trasplantados, con diferencias estadísticamente significativas en todas las dimensiones de las Escalas Específicas para Enfermedades Renales, excepto en el
Apoyo social, algo lógico al tener corregida su insuficiencia renal. De igual forma, los pacientes con TR presentaron mejores puntuaciones en todas las dimensiones del cuestionario genérico SF-36, algo que ya ha sido descrito por otros autores que encontraron una mejoría notoria en la CVRS tanto en las dimensiones específicas como genéricas al comparar los pacientes con $T R$ respecto a los pacientes en $\mathrm{HD}^{17,22,23}$, incluso llegando las puntuaciones obtenidas a ser similares a las de la población general24. Además del sexo, la edad o la comorbilidad, factores que se han asociado a peor CVRS en el paciente en HD, la probabilidad de padecer ansiedad y/o depresión, puede influir muy negativamente en peor percepción de la calidad de vida de estos pacientes, ya que casi la mitad de ellos son susceptibles de padecer alguna de estas alteraciones 0 ambas a la vez ${ }^{13}$, hecho que no suele ser tan frecuente en el paciente con $\mathrm{TR}^{25}$.

Cuando se compararon las puntuaciones entre ambos grupos de pacientes respecto a una de las variables influyentes en la CVRS en el paciente renal como es la comorbilidad, encontramos casi 2 puntos de diferencia en el ICC $(5,74 \pm 2,98$ en HD y $3,91 \pm 1,57$ en 
$T R, p<0,001)$. En los pacientes trasplantados el ICC se correlacionó con la edad, la Función física y el tiempo que llevaban los pacientes trasplantados, es decir mayor comorbilidad se asoció a mayor edad, a peor Función física y menos tiempo con TR. En cuanto a los pacientes en HD, mayor puntuación en el ICC se asoció también a mayor edad. Estos resultados concuerdan con lo publicado por otros autores, los cuales han mostrado que mayor comorbilidad se asocia a peor CVRS $^{26,27 .}$

Aunque las edades medias estaban igualadas, en los pacientes con TR la edad se asoció a Listado de síntomas/problemas y Situación laboral, en el cuestionario específico, y con la Función física, Dolor y Vitalidad del genérico SF-36. En los pacientes en HD la edad se asoció sólo a la dimensión Función física del SF-36. Estos resultados están en la línea de distintos estudios en los que la CVRS, en los que el aumento de edad se relaciona con más comorbilidad y con peores puntuaciones en la calidad de vida $13,26,27$.

Cuando se estudió el sexo y su influencia en la calidad de vida, encontramos que el sexo femenino influye de manera negativa, a la CVRS. En las pacientes con TR observamos una disminución de las puntuaciones en todas las dimensiones, existiendo diferencias significativas en el Listado de síntomas/problemas, Efectos de la enfermedad renal, Dolor y Vitalidad; mientras que en la población de HD sólo se encuentran diferencias significativas en la Función física. Estos resultados ya han sido mostrados por otros autores, en las que las mujeres tienen tener peor percepción de la CVRS que los hombres ${ }^{28,29}$.

La presencia de diabetes, además de su repercusión directa en la comorbilidad, se ha mostrado como un predictor de deterioro de la CVRS $^{30}$. En nuestros resultados hemos podido comprobar que tanto el paciente con TR como el paciente en HD diabético, presenta puntuaciones más altas en el ICC, con repercusión a su vez en la CVRS. Así, en el paciente trasplantado diabético puntuaron peor las dimensiones Carga de la enfermedad renal y Dolor. Por otra parte, en los pacientes en HD, sólo se observaron peores puntuaciones en la dimensión Rol emocional, aunque en este grupo de pacientes, el número de diabéticos fue muy pequeño como para establecer conclusiones. En cuanto al tiempo de TRS, en el paciente con TR observamos que este tiempo se asoció positivamente a las dimensiones Efectos de la enfermedad renal y Función física. Es decir, los pacientes experimentan una mejoría en algunas dimensiones de la CVRS con el paso del tiempo tras el $T R$, en la línea de lo aportado por otros autores ${ }^{31}$.

Por otro lado, los pacientes que llevaban más tiempo en $\mathrm{HD}$, presentaban peores puntuaciones en las dimensiones Efectos de la enfermedad renal, Función sexual, Sueño y Salud general, tal como han documentado diferentes estudios, que han relacionado la permanencia de mucho tiempo en diálisis con peor CVRS ${ }^{32}$.

Finalmente, para comprobar la influencia que pudiera tener el acceso vascular en la CVRS en los pacientes en HD, se compararon las puntuaciones del KDQOI-SF entre los pacientes que se dializaban con FAVa y los que lo hacían con un CATt, presentando estos últimos peores puntuaciones en general. En este sentido, algunos estudios han observado peores puntuaciones en algunas de las dimensiones en el KDQOL-SF en pacientes en HD con CATt, aunque sin resultados concluyentes ${ }^{13}$ y otros autores no han encontrado diferencias en cuanto a la calidad de vida entre los pacientes en HD que se dializaban mediante un catéter venoso central, en comparación con los pacientes que lo hacían a través de una fístula ${ }^{33}$.

Como principales limitaciones de este estudio podemos destacar que la muestra es pequeña debido a las dificultades para que los pacientes quisieran participar en el estudio por teléfono en plena pandemia por la COVID-19, y sobre todo, la imposibilidad de poder entrevistar personalmente a los pacientes en consulta, pues por teléfono algunos mostraban desconfianza con la entrevistadora. Otra limitación es la falta de referencias bibliográficas que analicen los mismos objetivos que nuestro estudio.

A la vista de nuestros resultados podemos concluir que: Emparejados por edad, sexo y comorbilidad, los pacientes con TR presentan mejor CVRS que los pacientes en HD, tanto en las Escalas específicas para la enfermedad renal como en las dimensiones del cuestionario genérico SF-36. Además, los pacientes con TR presentan menos comorbilidad.

En ambos grupos, los pacientes diabéticos presentaron más comorbilidad y peor CVRS. Mayor edad se asoció a más comorbilidad y peor CVRS. En los pacientes con TR, peor CVRS se asoció a más edad, sexo femenino, diabetes y comorbilidad. Sin embargo, a más tiempo trasplantados puntuaron mejor en las dimensiones Efecto de la enfermedad renal y Función física del cuestionario KDQOL-SF. En los pacientes en HD, los pacien- 
tes que llevan más tiempo en HD y los que se dializan con un CATt presentan peor CVRS. La influencia de la edad y el sexo femenino no parecen tan influyentes en la CVRS, al afectar ambas variables sólo a la dimensión Función física, ni tampoco la presencia de diabetes al afectar sólo al Rol emocional, debido posiblemente al tamaño de la muestra con pocas mujeres y pacientes diabéticos.

Estos resultados ponen de manifiesto que el TR debería ser el TRS de elección en todos los pacientes con ERCA, y si no existen contraindicaciones para el mismo, debe ser recomendado como opción de tratamiento, ya que es el que mejor CVRS aporta a estos pacientes.

Recepción: 05-12-20

Aceptación: 15-03-21

Publicación: 30-06-21

\section{Bibliografía}

1. Alcázar-Arroyo R, Orte-Martínez L, Otero-González A. Enfermedad renal crónica avanzada. Nefrología. 2008; (Supl 3):S3-6.

2. Górriz-Teruel JL, Otero-González A. Impacto socio sanitario de la enfermedad renalcrónica avanzada. Nefrología. 2008; (Supl 3):S7-15.

3. ERA-EDTA Registry Annual Report 2017. [consultado 7 may 2020]. Disponible en: https://eraedta-reg.org/files/annualreports/pdf/AnnRep2017. pdf.

4. King $H$, Aubert RE, Herman WH. Global Burden of Diabetes, 1995-2025: Prevalence, numerical estimates, and projections. Diabetes Care. 1998;21(9):1414-31.

5. Registro Español de Enfermos Renales. Informe de Diálisis y Trasplante 2018. [consultado 10 may 2020]. Disponible en: https://www.senefro.org/ contents/webstructure/SEN_2019_REER_modificada.pdf.
6. Guerrero-Riscos MA, Toro-Prieto FJ, BatalhaCaetano P, Salgueira-Lazo M, González-Cabrera F, Marrero-Robayna $S$, et al. Estudio ERCA. Características basales, evaluación de la aplicación de la información estructurada para la elección de tratamiento renal sustitutivo y evolución a un año de los pacientes incidentes en la consulta ERCA. Nefrología. 2019;39(6):563-82.

7. Arruda-Costa GM, Gomes Nascimento-Pinheiro MB, María-Madeiros S, de Oliveira-Costa RR, Santos-Cossi M. Calidad de vida en pacientes con insuficiencia renal crónica en hemodiálisis. Enfermería Global. 2016;15(43):59-73.

8. Vergara-Quintero $M$ del C. TRES CONCEPCIONES HISTÓRICAS DEL PROCESO SALUD-ENFERMEDAD. Hacia la Promoción de la Salud. 2007; (12):41-50.

9. Lizán-Tudela L. La calidad de vida relacionada con la salud. Aten Primaria. 2009;41(7):411-6.

10. Garrido-Blanco R, Arroyo-Priego E, Arana-Ruiz A, López-Zamorano M, Tierno-Tendero C, Crespo-Montero R. Calidad de vida y enfermedad renal crónica avanzada. Influencia del aclaramiento renal. Enferm Nefrol. 2018;21(4):359-67.

11. Ruiz-Parrado M del C, Gómez-Pozo M, Crespo-Garrido M, Tierno-Tendero C, Crespo-Montero R. Análisis de la calidad de vida del paciente en la etapa prediálisis. Enfermería Nefrológica. 2017;20(3):233-40.

12. Adell-Lleixà $M$, Casadó-Marín $L$, Andújar-Solé $J$, Solà-Miravete E, Martínez-Segura E, Salvadó-Usach T. Valoración de la calidad de vida relacionada con la salud en la enfermedad renal crónica terminal mediante un cuestionario de resultados percibidos por los pacientes. Enferm Nefrol. 2016;19(4):331-40.

13. Merino-Martínez RM, Morillo-Gallego N, Sánchez-Cabezas AM, Gómez-López V, Crespo-Montero R. Relación entre la calidad de vida relacionada con la salud y la ansiedad/depresión en pacientes en hemodiálisis crónica. Enferm Nefrol. 2019;22(3):274-83.

14. Costa-Requena G, Cantarell MC, Moreso F, Parramon $G$, Seron D. Calidad de vida relacionada con la 
salud en el trasplante renal: seguimiento longitudinal a 2 años. Med Clínica. 2017;149(3):114-8.

15. Dada-Ruiz D, Fernández-Aguirre C, Segura-González F, Carballo-Ruíz M. Indications and long term outcomes for solid organ transplant. Quality ol life in solid organ transplant recipients. Med Intensiva. 2008;32(6):296-303.

16. Fiebiger W, Mitterbauer C, Oberbauer R. Health-related quality of life outcomes after kidney transplantation. Health Qual Life Outcomes. 2004;2(1):2.

17. Tonelli M, Wiebe N, Knoll G, Bello A, Browne $S$, Jadhav $D$, et al. Systematic review: kidney transplantation compared with dialysis in clinically relevant outcomes. Am J Transplant Off J Am Soc Transplant Am Soc Transpl Surg. 2011;11(10):2093-109.

18. Pérez-Blancas C, Moyano-Espadero Ma C, Estepa-del Árbol M, Crespo-Montero R. Factores asociados a calidad de vida relacionada con la salud de pacientes trasplantados de riñón. Enferm Nefrológica. 2015;18(3):204-26.

19. Cirera-Segura F, Reina-Neyra M, Martín-Espejo $J$, Franco-Maldonado M. Diferencias entre el trasplante renal y otros tratamientos de la enfermedad renal crónica respecto a la calidad de vida. Seden. 2008;33. Disponible en: https://www.revistaseden.org/files/2021_1[2].pdf.

20. García F, López K, De Álvaro F, Alvárez-U de F, Alonso J, en representación del grupo CALVIDIA. Salud percibida en pacientes que comienzan tratamiento renal sustitutivo: validación preliminar de la versión española del KDQOL-SF. Nefrología 1998;18(Suppl 3):S66.

21. Sánchez-Cabezas AM, Morillo-Gallego N, Merino-Martínez RM, Crespo-Montero R. Calidad de vida de los pacientes en diálisis. Revisión sistemática. Enferm Nefrol. 2019 Jul-Sep;22(3):239-55.

22. Ortega F, Rebollo P, Bobes J, González MP, Saiz P. Interpretación de los resultados de la calidad de vida relacionada con la salud de pacientes en terapia sustitutiva de la insuficiencia renal terminal. Nefrología. 2000; 20(5):431-9.
23. Tamura $Y$, Urawa $A$, Watanabe $S$, Hasegawa $T$, Ogura T, Nishikawa K, et al. Mood Status and Quality of Life in Kidney Recipients after Transplantation. Transplantation Proceedings. 2018. doi:10.1016/j. transproceed.2018.03.077.

24. Costa-Requena G, Cantarell Aixendri MC, Rodriguez Urrutia A, Seron Micas D. Calidad de vida relacionada con la salud y trasplante renal: comparación con los valores poblacionales a los 6 meses postrasplante. 2014. Medicina Clínica. 142(9):393-6.

25. Moreno-Rubio F, Mora-Villarroel SL, Castelblanco-Toro C, Molina-López C, Ortiz-Varela LA. Trastorno emocional en el paciente renal trasplantado. Enferm Nefrol. 2016;19(2):147-53.

26. Ortega F, Rebollo P, Bobes J, González MP, Saiz P. Factores asociados a la calidad de vida relacionada con la salud (CVRS) de los pacientes en terapia renal sustitutiva (TRS). Nefrología. 2000;20(2);17151.

27. Cobo JL, Pelayo R, Ibarguren E, Aja A, Saenz de Buruaga $A$, Incera ME et al. Factores sociológicos y factores de vida relacionada con la salud en pacientes en hemodiálisis. Rev Soc Esp Enferm Nefrol. 2011;14(2):98-104.

28. Barbero $E$, Tejeda $E$, Herrera $C$, Montserrat $S$, Gascó $\mathrm{N}$, Junyent E. Estudio comparativo del estado físico, mental y percepción de calidad de vida relacionada con la salud de los pacientes en diálisis. Enferm Nefrol. 2016;19(1):29-35.

29. Albañil-Frías T, Ramírez-Moreno MC, Crespo-Montero R. Analysis of the quality of life in ambulatory hemodialysis patients and its relationship to the degree of dependence. Enferm Nefrol. 2014; 17(3):167-74.

30. Weber M., Faravardeh A., Jackson S., Berglund D., Spong R., Matas A.J., Ibrahim H.N. Quality of life in elderly kidney transplant recipients. J Am Geriatr Soc. 2014;62(10):1877-82.

31. Pérez San Gregorio MA, Martín Rodríguez A, Díaz-Domínguez R, Pérez-Bernal J. Evolución de la calidad de vida relacionada con la salud en los trasplantados renales. Nefrologia. 2007;27(5):619-26. 
32. Rebollo-Rubio A, Morales-Asencio JM, Pons-Raventos ME M-FJ. Review of studies on health related quality of life in patients with advanced chronic kidney disease in Spain. Nefrologia. 2015;35(1):92-109.

33. Pelayo-Alonso R, Cobo-Sánchez J, Reyero-López $M$, Sáenz de Buruaga-Perea A, Tovar-Rincón A, Alonso-Nates $\mathrm{R}$ et al. Repercusión del acceso vascular sobre la calidad de vida de los pacientes en tratamiento con hemodiálisis. Rev Soc Esp Enferm Nefrol. 2011;14(4):242-9.

Este artículo se distribuye bajo una Licencia Creative Commons Atribución-NoComercial 4.0 Internacional. https://creativecommons.org/licenses/by-nc/4.0/

\section{Open Access (C) (7)}

